\title{
Review of: "T-cell and antibody responses to first BNT162b2 vaccine dose in previously infected and SARS-CoV-2-naive UK health-care workers: a multicentre prospective cohort study"
}

\author{
Justyna Fert-Bober
}

Potential competing interests: The author(s) declared that no potential competing interests exist.

\begin{abstract}
Quality of adaptive immune responses following a single dose of COVID-19 vaccine in general population.
\end{abstract}

The COVID-19 pandemic has resulted in over 269 million cases and 5.3 million deaths worldwide as of December 2021, with even higher numbers due to continuous emerging variants, leading to large-scale disruption of societies. Although public health actions, such as lockdown measures, physical distancing, and improved hygiene practices, can reduce the likelihood of transmission, only the COVID-19 vaccination program has proven highly effective in reducing infection-related hospitalization and mortality in many demographic subgroups. At the beginning of the vaccination programs, many countries decided to prioritized vaccination of those at the highest risk of COVID-19 mortality and hospitalization, including older population in nursing home residents and individuals with increased probability of exposure to COVID patients (e.g., frontline health and social care workers). Furthermore, many countries decided to delay administration of second doses to 8-12 weeks rather than the shorter 3-weeks interval according to the manufactory's recommended dosing. Decision behind the delay of the second dose was justified by prioritization of the rapid administration of a single dose to a greater proportion of the population.

The main questions are whether the humoral and cellular immune responses following a single and a delay second dose of the BNT162b2 mRNA vaccine (tozinameran; developed by Pfizer-BioNTech) i) induces a similar level of protection against SARS-CoV-2 infection and ii) whether individuals with previous SARS-CoV-2 infection are better protected against SARS-CoV-2 or other variants of concern compared to vaccinated SARS-CoV-2 naïve individuals?

Vaccination with any viral protein antigens usually result in both a cellular (T- lymphocytes known as T-cells) and humoral (B- lymphocytes, known as B-cell) immune response capable of rapidly controlling replicating pathogens or inactivating their toxic components. Vaccine-induced B lymphocytes produce antibodies (e.g., IgG) against a toxin or a pathogen while CD8+ T lymphocytes may limit the spread of infectious agents by recognizing and killing infected cells or secreting specific antiviral cytokines, such as interferon gamma (IFNY), tumor necrosis factor (TNF $\alpha$ ). The SARS-CoV-2 messenger RNA (mRNA) vaccines BNT162b2 (Pfizer-BioNTech) and mRNA-1273 (Moderna) that encodes a SARS-CoV-2 spike protein induces a persistent immune response that protects against the real virus if enters our bodies. Both vaccines have 
each shown more than 90\% efficacy in preventing COVID-19 illness [1,2], however the longitudinal assessment of humoral immune responses in the previous SARS-CoV-2 infected verses SARS-CoV-2 naïve vaccinated individuals has not been documented well.

\section{B cell responses to mRNA vaccination}

A recent study by Adrienn Angyal et al. showed both the humoral immune and cellular response following a single and two doses of the BNT162b2 in a prospective cohort of healthcare workers recruited from five sites in the UK between December 9, 2020, and February 9, 2021 [3]. Within healthcare workers $(n=289)$ with serological evidence of previous infection, who received a single dose of vaccination $(n=145)$, the median IgG antibody titers against SARS-COV2 spike protein was approximately $7 \cdot 7$ times higher than post-vaccine concentrations in infection-naive individuals (median 270373 [IQR 203 461-535 188] AU/mL, $p<0.0001)$. The SARS-CoV-2-naive individuals after a single BNT162b2 dose $(n=119)$ were also protected with 3.3 times higher a spike IgG antibody response compared to pre-vaccine antibody concentrations (median 35001 [IQR 17 099-55 341] AU/mL vs median 10606 [IQR 4767-19 685] AU/mL, p<0.0001). Notably, following two doses of BNT162b2, SARS-CoV-2-naive healthcare workers $(n=25)$ had median antibody concentrations of 180904 (IQR 108 221-242 467) AU/mL, which were approximately 1.5 times lower than those following one dose in previously infected healthcare workers $(p<0.0001)$.

The magnitude of IgG antibody response after vaccination was then assessed in relation to age in participants with and without previous infection. Increasing age was associated with lower antibody titres (-0.0070 [ $\log 10] \mathrm{AU} / \mathrm{mL}$ per year; $p=0.0009)$, particularly in SARS-CoV-2 naïve individuals. The magnitude of antibody response after vaccination was negatively correlated with increasing age ( $r s=-0.38$, $\mathrm{p}<0.0001$ ), and was most notably decreased in those older than 60 years, whereas no such effect was observed in previously infected participants $(r s=0.014, p=0.87)$ or SARS-CoV-2 naïve individuals who had received two doses of the BNT162b2 ( $r s=-0.26, p=0.21)$. This observation was confirmed by Canaday et al. study that showed a faster decay of antibodies in persons 65 years or older, with lower anti-SARS CoV-2 Spike protein and neutralizing antibodies at 2-6 months post vaccination [4].

Considerable differences in amount (titres) of IgG antibodies were observed between participants who had previously been infected with SARS-CoV-2 and the time of their first vaccine dose. The paper reported that the titres of IgG antibodies against SARS-CoV-2 spike protein increased with time since first vaccination ( $r s=0.41, p<0.0001$ ), suggesting that a longer time interval between infection and the first vaccine dose was associated with greater boosting of antibody concentrations. The approach reported by Angyal et al. [3] was limited in number of participants however, similar results reported by Gallais et al.[5], showed two times higher IgG antibody response in previously SARS-CoV-2 infected individuals vaccinated at 7-11 months after they had been infected with SARS-CoV-2 virus [5]. Noteworthy, the Angyal et al. study included data on IgG antibody responses following BNT162b2 vaccine in infection-naive and previously infected individuals to seasonal human coronaviruses (alphacoronavirus: HCoV-229E, HCoVNL63, and beta-coronavirus: HCoV-HKU1, HCoV-OC43), as well as SARS-CoV and MERS-CoV. The group 
found that a single BNT162b2 dose elicited antibody response to SARS-CoV and MERS-CoV spike protein in both infection-naive and previously infected individuals $(p<0.0001)$. Respectively, the highest antibody response to both SARS-CoV and MARS-CoV, was recorded in individuals with single BNT162b2 dose in SARS-CoV-2-infected individuals, along with infection-naive individuals following two doses, followed by antibody response to a single dose in infection-naive individuals. Post-vaccine antibody concentrations were higher for HKU1 (median 29666 [IQR 12 454-48 789] AU/mL vs 14636 [7659-33344] AU/mL, $\mathrm{p}<0.0001$ ) and OC43 (median 108282 [50 278-175 835] AU/mL vs 48353 [24 037-102 143] AU/mL, $\mathrm{p}<0.0001$ ) respectively in those with previous SARS-CoV-2 infection than in infection-naive individuals [3]. These results suggest that SARS-CoV-2 mRNA-based vaccination enables the generation of robust humoral immunity.

A further interesting aspect reported in this paper is the ability of vaccination to protect against SARS-CoV2 variants of concern, such as lineage the alpha variant, initially reported in the UK (B.1.1.7)[6], beta variant, initially reported in South Africa (B.1.351), Delta (B.1.617.2 and AY lineages) initially reported in India [7] and Omicron (B.1.1.529 and BA lineages) variant, initially reported in South Africa which contain mutations in the viral spike protein [8]. In the Angyal et al. study, the authors examined IgG antibody responses to SARS-CoV-2 spike proteins to variants of concern using eighter a surrogate neutralisation or a live virus neutralisation assay. The results showed similar titers of the antibody across infection-naive and previously infected individuals to spike glycoproteins from the recently identified lineages [3]. Importantly, a single BNT162b2 dose resulted in substantially increased neutralizing IgG antibody activity against all of the viral variants tested. A higher anti-SARS-CoV-2 IgG antibody boost was detected in healthcare workers with previous SARS-CoV-2 infection than in infection-naive healthcare workers (postvaccine median 29.7 [14.6-37.4] units per $\mathrm{mL}$ verses 1.9 [1.4-3.2] units per $\mathrm{mL}, \mathrm{p}=0.0006$ ), with a significant correlation between surrogate and live virus neutralization assays for both virus lineages $B$ $(r s=0.91, p<0.0001)$ and beta $(r s=0.78, p<0.0001)$. This suggests that vaccination following prior infection substantially increases the breadth of cross-reactive viral neutralizing antibodies. Similarly, Lustig et. al. showed that with one BNT162b2 vaccine dose there is an increased in neutralizing activity by two orders of magnitude against all variants tested (B.1.1.7 or alpha, B.1.351 or beta, P.1 and gamma) in previously infected healthcare workers [9]. What remains to be investigated is whether the effects of a second vaccine dose on neutralizing activity against variants of concern in naïve persons and previously SARSCoV-2-infected individuals. Nevertheless, the susceptibility of the virial variant strains to neutralization elicited by the BNT162b2 vaccine supports mass immunization as a central strategy to end the COVID-19 pandemic globally.

\section{T cell responses to mRNA vaccination}

Alongside antibodies, the immune system produces a battalion of T-cells that also can target viruses. Prior studies have shown that memory B-cell responses tend to be short lived after infection with SARS-CoV$1[10,11]$. In contrast, memory T-cell responses can persist for many years. A recent study showed that 
SARS-CoV-2-specific memory CD4+ and CD8+ T cell responses are sustained in COVID-19 convalescent individuals for 10 months regardless of disease severity [12]. Interestingly, the SARS-CoV-2 infection status had a major impact on the profile of the memory CD4+ and CD8+ T cells in response to BNT162b2 mRNA vaccine and markedly enhances adaptive immune responses.

As discussed in the Angyal's manuscript, the T-cell response assessed by changes in the IFNY concentration in the peripheral blood mononuclear cells (PBMCs) (measured by (enzyme-linked immunospot (ELISpot) assay) increased in the SARS-CoV-2-naive individuals after a single vaccine dose (median 55 [IQR 24-132] SFUs/106 PBMCs, p<0.0001). The response was lower than T-cell responses in the previously infected healthcare workers (median 93 [IQR 48-161] SFUs/106 PBMCs) before vaccination. A second vaccine dose in the infection-naive individuals further increased the T cell response and match the level of the T-cell response to previously infected individuals after a single dose of vaccination (median 284 [IQR 150-461] SFUs/106 PBMCs, p<0.0001). In fact, a 5.2 times higher peak of the T cell response was detected in the individuals previously infected compared to infection-naïve individuals after a one dose of vaccine. These findings have been confirmed by other groups and are useful to consider in any discussions around vaccination strategy [13]. As expected, there was no significant change in T cell response to $\mathrm{N}$ protein (a measure of immunity to natural infection) in either group.

The Angyal's et al., have additionally compared the spectrum of cytokine responses in SARS-CoV-2-naive $(n=31)$ or previously infected individuals $(n=31)$ and revealed that BNT162b2 vaccine alter the profile of cytokine production. The IFN- $y$ and TNFa protein expression levels were higher and correlated with S1 and S2 CD4+ T-cell proliferation in previously infected individuals. Unfortunate, the intracellular cytokine staining was performed only on the vaccinated individuals, and as presented the vaccination increase the T cell responses, making it not possible to directly compare the cytokine expression level between vaccinated and non-vaccinated individuals. The study also included functional expression analysis, where overall similar profile of functionality in both CD4+ and CD8+ T cells were showed. One limiting factor of the study were the low numbers of individuals included, especially the for CD8+T cells response ( $n=4$ and $\mathrm{n}=3$ previously infected) therefore there might have been a bias towards the selection of individuals with stronger pre-existing immune capacity.

It is well appreciated that the vaccine-induced T-cell responses to four separate peptide pools spanning the breadth of the spike protein (spike1-330, spike321-645, spike636-690, and spike950-1273;) were identified in both previously infected and infection-naive individuals. Responses in individuals with previous infection were characterized by the dominance of responses to spike1-330 and spike636-690 in some individuals, compared to a more balanced response across all peptide's pools follow by a single BNT162b2 dose. This phenomenon may suggest boosting of sub-dominant responses towards epitopes that were previously below the limits of detection in previously infected individuals. This finding should be emphasized as broadening of the T-cell response with vaccination following natural infection provides substantially enhanced immune protection. 


\section{Discussion}

The results shown by Angyal et al. that BNT162b2 vaccination elicits a strong IgG antibody response against SARS-CoV-2 as well as other SARS-CoV or MARC-CoV variants although with a markedly lower response. The second vaccination dose induced the typical booster increase in IgG antibody as expected for a nonimmune population. The median responses to SARS-CoV-2 and SARS-CoV were 7.7 times and 6.7 times greater the pre vaccination levels, respectively. In individuals previously infected the antibody response increased 15 times higher compared to naïve individuals [3]. The reasons for the strong enhancement of humoral immunity by previous natural infection are yet to be determined.

The author of "T-cell and antibody responses to first BNT162b2 vaccine dose in previously infected and SARS-CoV-2-naive UK health-care workers: a multicentre prospective cohort study" also observed a progressive increase in the percentage of $C D 4+C D 8+T$ cells response following BNT162b2 vaccination with domination of CD4+ response in previously infected individuals, in contrast to infection-naive individuals who after BNT162b2 vaccination showed balanced CD4+ and CD8+ response. These observations supports that there is potential protection against multiple viral variants of concern within previously infected and single-vaccinated people. As explain by the author, broadening of the T-cell response could lead to more effective protection against emerging SARS-CoV-2 variants with antibodyescape mutations. Therefore, increasing the proportion of the population immunized with current safe and effective authorized vaccines should remain a key strategy to minimize the emergence of new variants. One major limitation of this study includes the fact that $80 \%$ of the participants were young, healthy females. Generally, among adults of reproductive ages, females have greater antibody responses than males, higher IgG levels, and higher B cell numbers [14-16]. Previously, it has been shown that adult females have higher antibody responses to diverse vaccines against, influenza, hepatitis B, yellow fever, rabies, herpes, and smallpox viruses [17-20]. The same pattern can be seen after BNT162b2 vaccination [21]. Furthermore, changes in the humoral immune response during aging has been described and associated with an increased susceptibility to infectious diseases in older adults [22,23]. Notably, study by Collier et al., reported vaccine response to be lower in older population [23]. Furthermore, the effect of mutation only in the SARS-CoV-2 spike protein was considered. Mutations outside the spike gene could also affect viral replication and host immune response. In summary, after vaccination, infection-naive individuals developed antibody responses similar to those seen in naturally infected persons before vaccination, however vaccination is required to markedly enhance adaptive immune responses in both groups, previously infected and SARS-CoV-2-naive. The expedited second vaccine dose administration is advisable to deliver particularly to SARS-CoV-2-naive and older population, particularly where variants of concern are circulating.

\section{Reference}

1. Baden LR, El Sahly HM, Essink B, et al. Efficacy and Safety of the mRNA-1273 SARS-CoV-2 Vaccine. 
The New England journal of medicine. 2021 Feb 4;384(5):403-416.

2. Polack FP, Thomas SJ, Kitchin N, et al. Safety and Efficacy of the BNT162b2 mRNA Covid-19 Vaccine. The New England journal of medicine. 2020 Dec 31;383(27):2603-2615.

3. Angyal A, Longet S, Moore SC, et al. T-cell and antibody responses to first BNT162b2 vaccine dose in previously infected and SARS-CoV-2-naive UK health-care workers: a multicentre prospective cohort study. The Lancet Microbe. 2021 2021/11/09/.

4. Canaday DH, Oyebanji OA, Keresztesy D, et al. Significant reduction in humoral immunity among healthcare workers and nursing home residents 6 months after COVID-19 BNT162b2 mRNA vaccination. medRxiv. 2021:2021.08.15.21262067.

5. Gallais F, Gantner P, Bruel T, et al. Anti-SARS-CoV-2 Antibodies Persist for up to 13 Months and Reduce Risk of Reinfection. medRxiv. 2021:2021.05.07.21256823.

6. Rambaut A, Holmes EC, O'Toole Á, et al. A dynamic nomenclature proposal for SARS-CoV-2 lineages to assist genomic epidemiology. Nature microbiology. 2020 2020/11/01;5(11):1403-1407.

7. Mlcochova P, Kemp SA, Dhar MS, et al. SARS-CoV-2 B.1.617.2 Delta variant replication and immune evasion. Nature. 2021 2021/11/01;599(7883):114-119.

8. Dubey A, Choudhary S, Kumar P, et al. Emerging SARS-CoV-2 Variants: Genetic Variability and Clinical Implications. Current Microbiology. 2021 2021/12/14;79(1):20.

9. Lustig $Y$, Nemet I, Kliker L, et al. Neutralizing Response against Variants after SARS-CoV-2 Infection and One Dose of BNT162b2. The New England journal of medicine. 2021 Jun 24;384(25):2453-2454.

10. Channappanavar R, Fett C, Zhao J, et al. Virus-specific memory CD8 T cells provide substantial protection from lethal severe acute respiratory syndrome coronavirus infection. Journal of virology. 2014 Oct;88(19):11034-44.

11. Tang F, Quan Y, Xin ZT, et al. Lack of peripheral memory B cell responses in recovered patients with severe acute respiratory syndrome: a six-year follow-up study. J Immunol. 2011 Jun 15;186(12):72648.

12. Jung JH, Rha MS, Sa M, et al. SARS-CoV-2-specific T cell memory is sustained in COVID-19 convalescent patients for 10 months with successful development of stem cell-like memory T cells. Nat Commun. 2021 Jun 30;12(1):4043.

13. Reynolds CJ, Pade C, Gibbons JM, et al. Prior SARS-CoV-2 infection rescues B and T cell responses to variants after first vaccine dose. Science (New York, NY). 2021 Apr 30.

14. Fink AL, Klein SL. The evolution of greater humoral immunity in females than males: implications for vaccine efficacy. Curr Opin Physiol. 2018 Dec;6:16-20.

15. Klein SL, Flanagan KL. Sex differences in immune responses. Nature reviews Immunology. 2016 Oct;16(10):626-38.

16. Klein SL, Marriott I, Fish EN. Sex-based differences in immune function and responses to vaccination. Trans R Soc Trop Med Hyg. 2015 Jan;109(1):9-15.

17. Furman D, Hejblum BP, Simon N, et al. Systems analysis of sex differences reveals an 
immunosuppressive role for testosterone in the response to influenza vaccination. Proceedings of the National Academy of Sciences of the United States of America. 2014 Jan 14;111(2):869-74.

18. Voigt EA, Ovsyannikova IG, Kennedy RB, et al. Sex Differences in Older Adults' Immune Responses to Seasonal Influenza Vaccination. Front Immunol. 2019;10:180.

19. Shohat T, Green MS, Nakar O, et al. Gender differences in the reactogenicity of measles-mumpsrubella vaccine. Isr Med Assoc J. 2000 Mar;2(3):192-5.

20. Andersen A, Bjerregaard-Andersen M, Rodrigues A, et al. Sex-differential effects of diphtheriatetanus-pertussis vaccine for the outcome of paediatric admissions? A hospital based observational study from Guinea-Bissau. Vaccine. 2017 Dec 15;35(50):7018-7025.

21. Ebinger JE, Fert-Bober J, Printsev I, et al. Antibody responses to the BNT162b2 mRNA vaccine in individuals previously infected with SARS-CoV-2. Nat Med. 2021 Jun;27(6):981-984.

22. Frasca D, Diaz A, Romero M, et al. Age effects on B cells and humoral immunity in humans. Ageing Res Rev. 2011 Jul;10(3):330-5.

23. Collier DA, Ferreira IATM, Kotagiri P, et al. Age-related immune response heterogeneity to SARS-

CoV-2 vaccine BNT162b2. Nature. 2021 2021/08/01;596(7872):417-422. 